\title{
Online low-fidelity ward simulation to complement learning of clinical pharmacy: a response to the COVID-19 pandemic.
}

\author{
Jamil, N., Jamludin, N.A., Chee, A.C.S., Lian, L.S., Hisham, S.A. \\ University of Cyberjaya, Malaysia
}

\section{Background:}

During COVID-19 pandemic, clinical pharmacy attachments were discouraged in order mitigate the spread of infection. Low-fidelity simulation allows experience of approximate actual environment of learning with lower resource utilisation. The use of online low-fidelity ward simulation to complement learning of clinical pharmacy was explored as adaptation to missed experiential learning.

\section{Methods:}

A low-fidelity simulation exercise that modelled activities of a clinical pharmacist practicing in the medical ward was designed. 68 final year pharmacy students from the University of Cyberjaya were assigned to a simulated 5-bedded medical ward on Microsoft Teams. Students undertook various core tasks of the pharmacist including medical reconciliation, participating in ward rounds, proposing pharmaceutical care interventions, documenting in medical records and medication counselling. An online pre- and post- survey was conducted to assess anticipated and actual usefulness of the designed activity. Open text answer regarding main activity contributing to increased confidence was permitted.

\section{Results:}

68 students completed the pre- and 55 students completed the post- simulation survey. Matched-pair analysis were conducted for complete pairs. Overall, students perceived improved skills in clinical pharmacy care provision except in patient communication and resolving drug-related problems. Emergent themes from open text answers were "ward rounds with simulated physician" "identifying pharmaceutical care interventions and recommending solutions" "answering physician inquiries" and "medication reconciliation" The answers demonstrate appreciation of interaction and achievement of tasks in building confidence. $47 \%$ students preferred interactive face-to-face as simulation, $38 \%$ preferred interactive online simulation and $13 \%$ had no preference. Only $9 \%$ reported experiencing moderate technological difficulties.

\section{Conclusions:}

Online ward simulation was perceived useful to gain knowledge and skills in pharmaceutical care provision towards medical patients. Learning activities should incorporate interaction with other simulated healthcare providers and allow a sense of achievement.

Keywords: Online learning, ward simulation, low-fidelity, ward pharmacy 